\title{
Short-Term Load Forecasting using Statistical Methods: A Case Study on Load Data
}

\author{
Shaik Dai Haleema \\ Electrical Engineering Department, \\ V. R. Siddhartha Engineering College, \\ Vijayawada, Andhra Pradesh, India.
}

\begin{abstract}
This paper presents the study of Moving Averages (MA), Autoregressive Moving Averages (ARMA) and Kalman Filter (KF) techniques for load forecasting. The data considered was Andhra Pradesh State electricity demand (MW) at every 15 minutes of $18^{\text {th }}$ May 2014. For the time series data both the methods ARMA and Kalman Filter techniques are used to predict and forecast the load. The results indicated that Kalman Filter gives better load forecasting as compared to ARMA in terms of less measurement of error using Mean Absolute Percentage Error (MAPE).
\end{abstract}

Keywords - Load forecasting, Statistical methods, Autoregressive Moving Average, Kalman Filter, and Mean Absolute Percentage Error (MAPE).

\section{INTRODUCTION}

Prediction of future events and conditions is called forecast and the act of making such predictions is called forecasting. Generally, in sectors like business and management forecasting plays a major role to provide information regarding the future environment [1]. But in Power systems load forecasting plays a vital role in energy management system. Precise load forecasting remains a great challenge due to data measurement and transmission problems, there may be bad data which deviate from real values and affects the precision of load forecasting results. The system operator uses the load forecasting result as a basis for off line network analysis to determine the vulnerability of the system else corrective actions should be prepared such as power trading, load shedding, power purchase and bringing peak units online. Load forecasting techniques are classified into long term, medium term and short-term load forecasting.

Load forecasts can be divided into three categories: short-term forecasts which are usually from one hour to one-week, medium forecasts which are usually from a week to a year, and long-term forecasts which are longer than a year. The forecasts for different time horizons are important for different operations within a utility company. The short-term forecast considers weather data, social events, holidays, festivals and other load data. The medium- and long-term forecasts take into account the historical load and weather data, the number of customers in different categories and industries.

Short-term load forecasting (STLF) has become increasingly important since the rise of competitive energy markets. The information obtained from short term load forecasting is vital to operational of dispatch center for dispatching the load economically. It also helps to estimate load flows, make decisions that can prevent overloading and reduce the equipment failures. Consequently, hourly and daily forecasts up to a few days ahead are of primary interest in everyday system operations.

Current methods for STLF may be classified into two categories - statistical methods (like similar- day, exponential smoothing, regression, and time series methods and methods based on artificial intelligence techniques (like neural networks, fuzzy logic, expert systems, and support vector machines).

The load forecasting can be estimated using statistical approaches. Statistical methods predict the current load value by using a mathematical combination of the previous loads and/or previous or current values of exogenous factors, e.g., weather and social variables. Although of statistical nature, they allow some physical interpretation making understanding load behavior easier. Regression is one of the widely used statistical approach employ to model a relationship of load consumption and other factors. Time series methods are based on the assumption that the data have an internal structure, such as autocorrelation, trend or seasonal variation. Moving Average, Autoregressive Moving Average (ARMA) and Kalman Filter techniques are used to predict and estimate the load [2].

According to [3], medium term forecast of 4-10 years is highly essential for planning the size of power plants, construction and installation of the equipment in power plants and for the addition of new transmission and distribution facilities. STLF using time series analysis is carried out for various load data by using AR, ARMA, and ARIMA techniques. MAPE is calculated for all the three techniques $[4,8,9]$. The load is forecasted by using Moving Average technique [5]. Hourly loads of weekdays, as well as, weekends and public holidays forecasting is also carried out [7]. Short term load forecasting by using Kalman Filter and ANN methods are performed and compared $[11,13]$.

This paper is presented in five parts, starting with an introduction highlighting the need for short term load forecasting. The subsequent sections review the different statistical methods, the application of these methods to Andhra Pradesh State System demand (MW) data taken day load demand of $18^{\text {th }}$ May 2014. Comments on results, concluding remarks and future scope have been presented.

\section{TIME SERIES ANALYSIS}

In time series analysis time series data is used. Time series data is a set of observations on the value that a variable takes at different times. Such a data is collected at regular interval of 
times, such as hourly, daily, weekly, monthly, quarterly, or annually for forecasting the future demand. Least square estimation using linear regression analysis is not a good technique for short term load forecasting because the Mean Square Error (MSE) is more and the accuracy is low.

Time series analyses are of different types, Moving Average (MA), Autoregressive (AR), Autoregressive Moving Average (ARMA), Autoregressive Integrated Moving Average (ARIMA), and Seasonal Autoregressive Integrated Moving Average (SARIMA). All the methods have its own procedures for forecasting the future demand.

\section{A. The Autoregressive(AR) Process}

It is similar to linear regression model, as the name itself indicates that its dependent and independent variables are different. In an AR model its independent variables are time lagged values of its dependent variables. The regression analysis depends on the order of the AR process. The order of AR denoted as ' $p$ ', the current value of the time series $Y_{t}$ is expressed linearly in terms of its ' $p$ ' previous values $\left[Y_{t-1}, Y_{t-2}, \ldots \ldots \ldots, Y_{t-p}\right]$ and a random noise $\varepsilon_{t}$.

The Autoregressive equation of order ' $p$ ' is denoted as,

$$
Y_{t}=\mu+\phi_{1} Y_{t-1}+\phi_{2} Y_{t-2}+\ldots \ldots \ldots+\phi_{n} Y_{t-p}+\varepsilon_{t}
$$

Here ' $\mu$ ' a constant mean, and $\phi_{1}, \phi_{2}, \ldots \ldots . ., \phi_{n}$ are AR coefficients.

By introducing the backshift operator $B$ that defines $Y_{t-1}=B Y_{t}$, and consequently $Y_{t-m}=B_{m} Y_{t}$, equation (1) can be written in the form of

$$
\phi(B) Y_{t}=\varepsilon_{t}
$$

Where

$$
\phi(B)=1-\phi_{1} B-\phi_{2} B^{2}-\ldots \ldots \ldots-\phi_{p} B^{p}
$$

\section{B. The Moving Average(MA) Process}

Simple Moving Average is the most basic of the Moving Averages used for short term load forecasting. The Moving Average is also defined as rolling average or running average. It is a calculation to analyze data points by creating the series of averages of different subsets of the full data set, which depends on the order of the MA process. The order of MA is denoted as ' $\mathrm{q}$ '. When Moving Average is combined with Autoregressive process, the current value of the time series $Y_{t}$ is expressed linearly in terms of current and previous ' $q$ ' values of the white noise series $\left[\varepsilon_{t}, \varepsilon_{t-1}, \varepsilon_{t-2}, \ldots \ldots \ldots, \varepsilon_{t-q}\right]$. The noise series is collected from forecast errors or residuals when load observations are available.

The Moving Average equation of order ' $\mathrm{q}$ ' is denoted as,

$$
Y_{t}=\varepsilon_{t}-\theta_{1} \varepsilon_{t-1}-\theta_{2} \varepsilon_{t-2}-\ldots \ldots \ldots-\theta_{n} \varepsilon_{t-q}
$$

Where $\theta_{1}, \theta_{2}, \ldots \ldots \ldots, \theta_{n}$ are MA coefficients.

Similarly, in the application of backshift operator on the white noise series the equation (3) can be expressed as,

$$
\phi(B) Y_{t}=\theta(B) \varepsilon_{t}
$$

\section{Where}

$$
\theta(B)=1-\theta_{1} B-\theta_{2} B^{2}-\ldots \ldots \ldots-\theta_{p} B^{p}
$$

C. The Autoregressive Moving Average(ARMA) Process ARMA process depends on Box-Jenkins [4,6] models. It is a combination of both AR and MA models, which can be described by a series of equations. The process depends on the order of AR and MA models denoted as (p, q), where ' $p$ ' and ' $q$ ' are the order of AR and MA process. In this ARMA process, the current value of time series $Y_{t}$ is expressed linearly in terms of its previous ' $\mathrm{p}$ ' values $\left[Y_{t-1}, Y_{t-2}, \ldots \ldots \ldots ., Y_{t-p}\right]$ of AR model, current and previous ' $\mathrm{q}$ ' values of a white noise $\left[\varepsilon_{t}, \varepsilon_{t-1}, \ldots \ldots \ldots, \varepsilon_{t-q}\right]$ of MA model.

The order of ' $p$ ' and ' $q$ ' were defined by the Partial Auto Correlation Function (PACF) and Auto Correlation Function (ACF) of the given time series data. Understanding the PACF and ACF is very important to identify which order to be used for ' $p$ ' and ' $q$ '. The thumb rule is order of ' $p$ ' taken from PACF and ' $q$ ' using ACF plots respectively.

ARMA (p, q) defines an Autoregressive Moving Average process of order ' $\mathrm{p}$ ' and ' $\mathrm{q}$ ' and the equation is expressed as, $Y_{t}=\mu+\phi_{1} Y_{t-1}+\phi_{2} Y_{t-2}+\ldots \ldots \ldots+\phi_{n} Y_{t-p}+\varepsilon_{t}+\theta_{1} \varepsilon_{t-1}+\theta_{2} \varepsilon_{t-2}+\ldots \ldots \ldots+\theta_{n} \varepsilon_{t-q}$

Where $\Phi$ and $\theta$ are coefficients of ARMA. ARMA model:

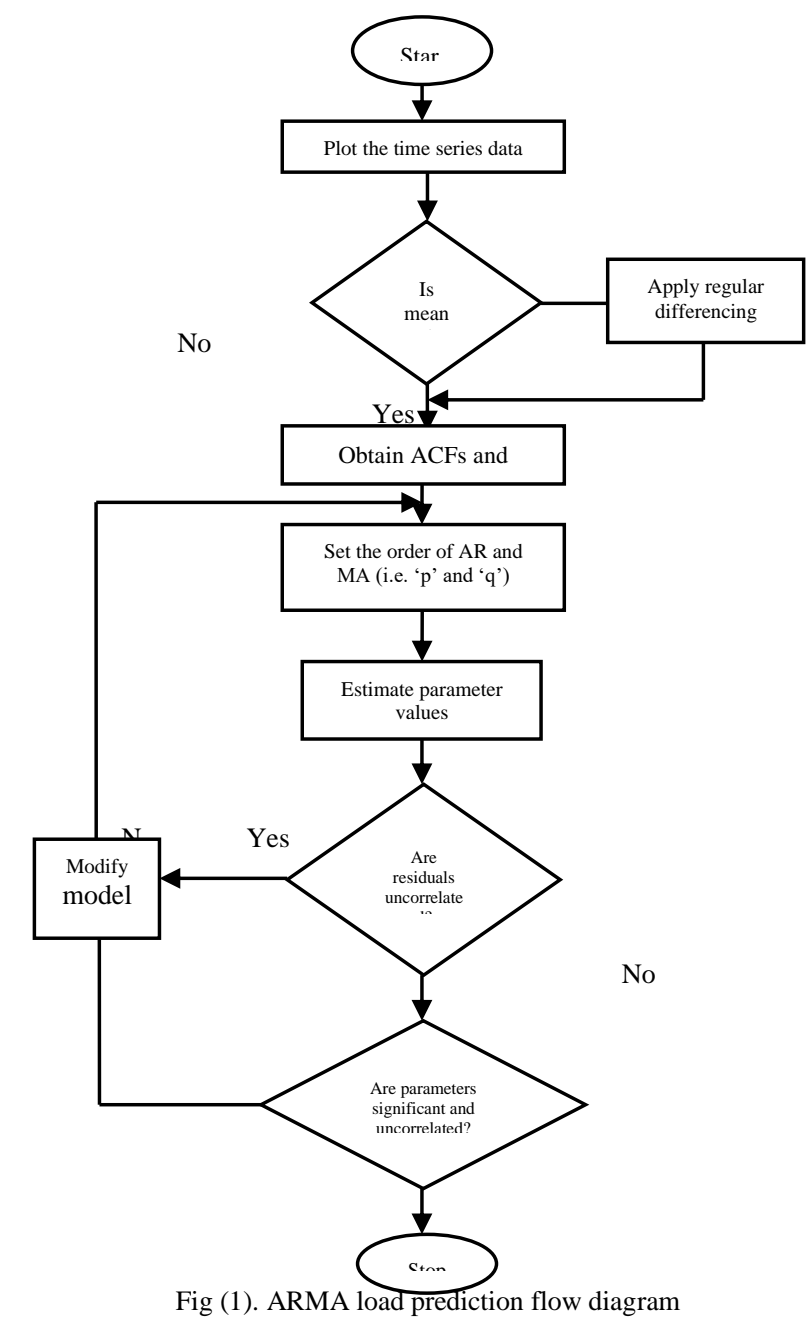

Algorithm steps to be followed for prediction based on 


\section{THE KALMAN FILTER}

Kalman Filter is one of the optimal filters to predict $Y_{t+1}$ from the prediction of $\mathrm{Y}_{\mathrm{t}}$. It is a linear minimum variance of error filter and is the best linear filter over all linear filters [12]. The time series approach has been extensively employed in dealing with the load forecasting problem in view of the relative simplicity of the model forms. However, this method tends to ignore the statistical information about the load data which may regularly be available, this may lead to improved load forecasts if utilized properly. In ARMA model load forecasting problem is not much simple and those difficulties may be avoided in some situations if Kalman Filtering techniques are used.

It is a recursive optimal estimator. It defines instant load forecast technique, because it considers the immediate samples for prediction [14,15]. The basic Kalman Filter algorithm includes two stages of equations at every instant of time. They are Time update equations and Measurement update equations. In general, KF uses current prediction error and current system demand data provided by data acquisition system to estimate the next state vector. The Time update equations are named as Predictor equations, while Measurement update equations are called Corrector equations. The final estimation algorithm of $\mathrm{KF}$ resembles that of a Predictor-Corrector algorithm for solving numerical problems $[13,16]$.

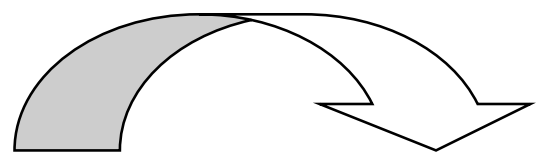

Time Update

Measurement Update ("Predict")

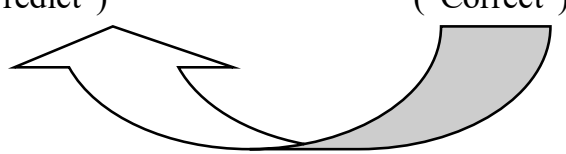

State Estimation Equations:

$$
\begin{aligned}
& Y_{t}=F Y_{t-1}+B U_{t-1}+w_{t-1} \\
& Z_{t}=H Y_{t}+v_{t-1}
\end{aligned}
$$

Predict Equations:

$$
\begin{aligned}
& \hat{Y}_{t}^{-}=F \hat{Y}_{t-1} \\
& P_{t}^{-}=F P_{t-1} F^{T}+Q
\end{aligned}
$$

Correct Equations:

$$
\begin{aligned}
& K_{t}=P_{t}^{-} H^{T}\left(H P_{t}^{-} H^{T}+R\right)^{-1} \\
& \hat{Y}_{t}=\hat{Y}_{t}^{-}+K_{t}\left(Z_{t}-H \hat{Y}_{t}^{-}\right) \\
& P_{t}=\left(I-K_{t} H\right) P_{t}^{-}
\end{aligned}
$$

Where

$\mathrm{Y}_{\mathrm{t}}$ is a system states of order $\mathrm{nx} 1, \mathrm{~F}$ is $\mathrm{nxn}$ state transition matrix, $Z_{t}$ is measurement vector of $m \times 1$ order, $H$ is output matrix of order mxn, $\mathrm{w}_{\mathrm{t}-1}$ is an uncorrelated white noise, $\mathrm{v}_{\mathrm{t}-1}$ is a mx1 uncorrelated white noise and having zero mean, $\mathrm{K}_{\mathrm{t}}=$ Kalman gain at time $t, \hat{Y}_{t}^{-}=$priory state estimate at previous time interval $(\mathrm{t}-1), \hat{\mathrm{Y}}_{\mathrm{t}}=$ posteriori state estimate at time $\mathrm{t}, \mathrm{P}_{\mathrm{t}}^{-}=$ priori estimate error covariance at previous time interval, $\mathrm{P}_{\mathrm{t}}=$ posteriori error covariance, $\mathrm{Q}=$ process noise, $\mathrm{R}=$ measurement noise.

To enhance the convergence characteristics of Kalman Filter, there must be smart preference of the priori estimate state $\hat{\mathrm{Y}}_{0}$ and its covariance error $\mathrm{P}_{0}$.

Assumptions made for convergence of Kalman Filter applying constant velocity model for predict and correct equations are:

a) State transition matrix $F$ is a constant marix.

b) $\mathrm{Q}$ value depends on actual characteristics of the past information of process noise.

c) $\mathrm{R}$ is a measurement noise, which is taken as an absolute value of 1 .

d) $\mathrm{H}$ is a constant matrix based on the constant velocity method.

e) $Z_{t}$ represents the optimal estimated load at a particular instant of time $t$.

Algorithm steps to be followed for prediction based on Kalman Filter model

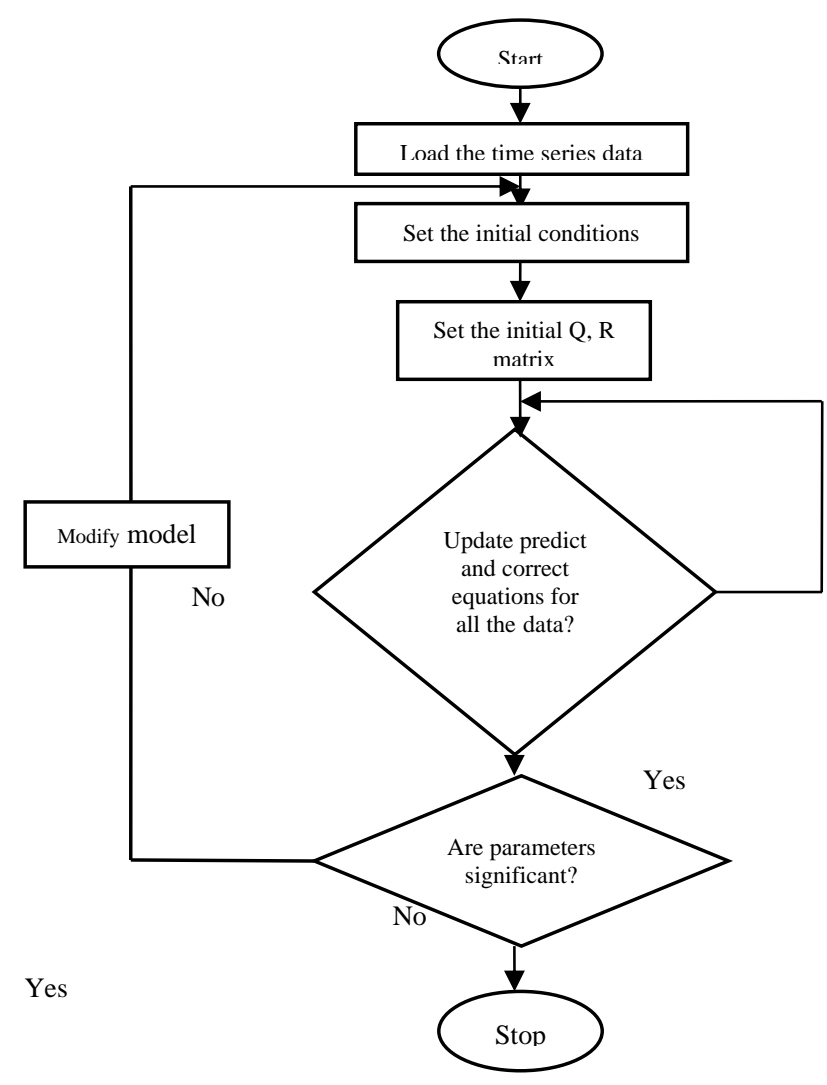

Fig (2). Kalman Filtering load prediction flow diagram

\section{RESULT \& DISCUSSION}

In this paper the data is collected from Andhra Pradesh State electricity at every 15 minutes of $18^{\text {th }}$ may 2014-day load demand (MW). In the current scenario, the major system operators consider the previous immediate similar day system demand (MW) curve as the reference demand load forecast. In this, the short-term load forecasting is estimated using ARMA and $\mathrm{KF}$ algorithms. 


\section{A. ARMA}

Short term load forecasting using ARMA technique was presented. From the ACF and PACF plots given in Fig (3) the order for AR model is 2 and MA model is 1 and the load demand was forecasted using ARMA $(2,1)$ and the results are presented in Fig (5) which is a zoomed form of Fig (4).

Blue line is an original data and the red line indicates the forecasted data, where it is observed that the load forecasting has been estimated accurately with some measurement error.

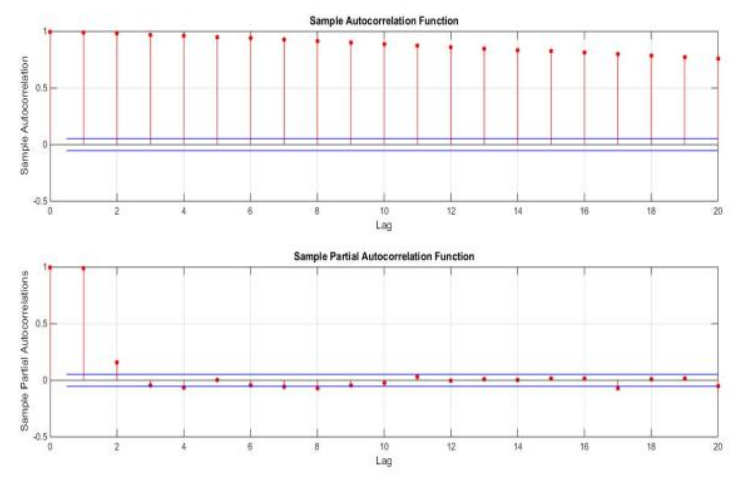

Fig (3). ACF and PACF plots

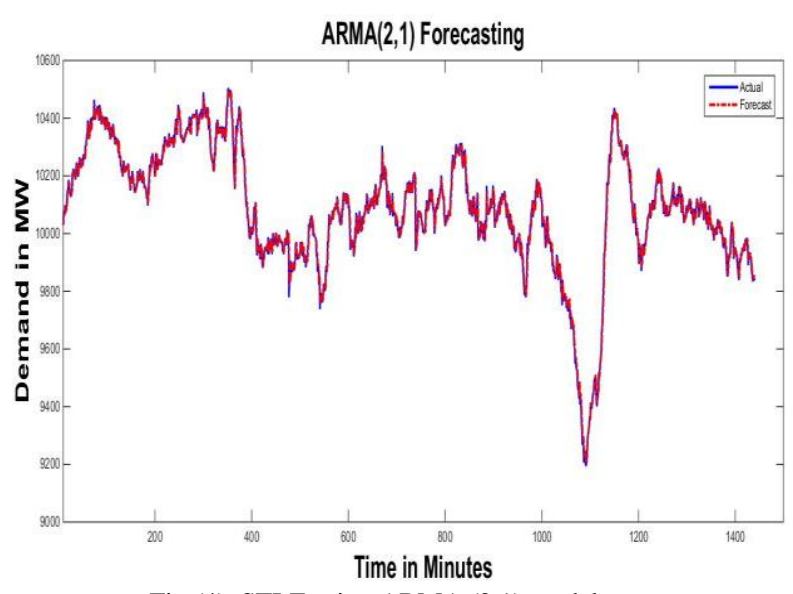

Fig (4). STLF using ARMA $(2,1)$ model

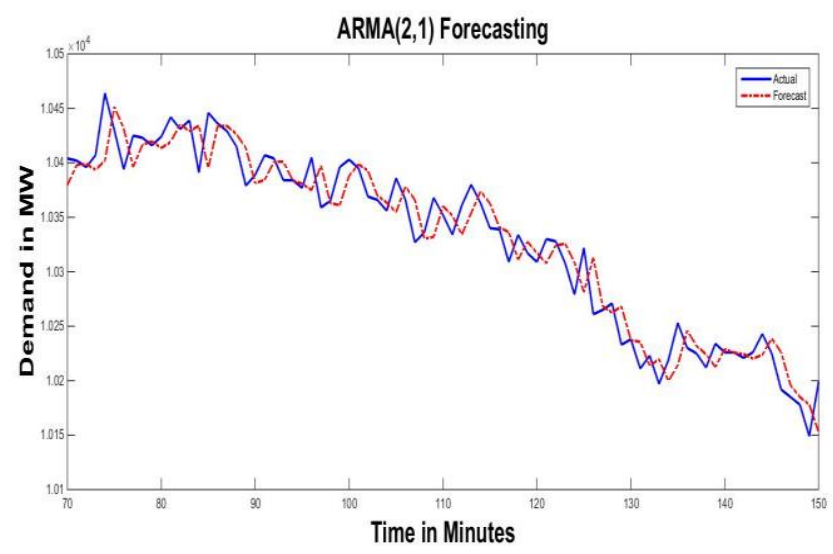

Fig (5). STLF ARMA $(2,1)$ zoomed plot

\section{B. Kalman Filter}

Short term load forecasting using Kalman Filter was presented and the results are presented in Fig (6) and Fig (7) is zoomed form of Fig (6). From the figures it was observed that load forecasting has been estimated accurately with some forecasting error. Fig (7) gives the clean observation of error between actual value and the forecasted value.

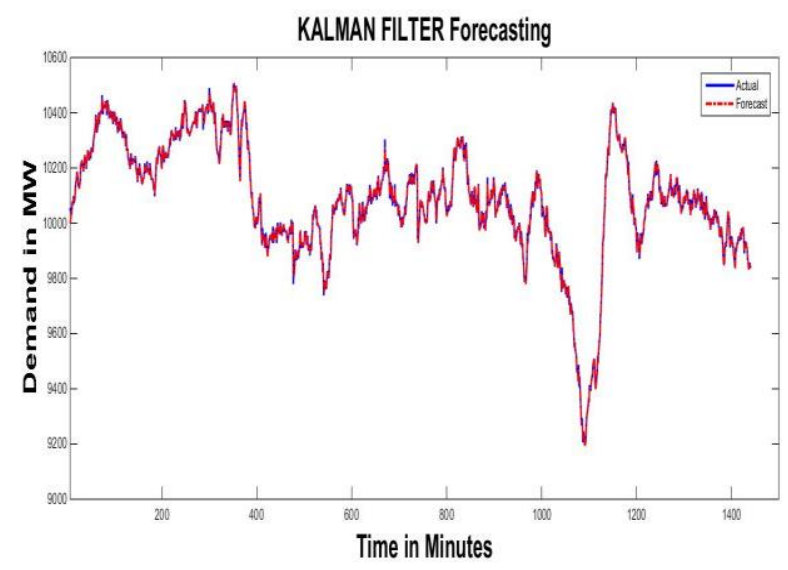

Fig (6). STLF using Kalman Filter Algorithm

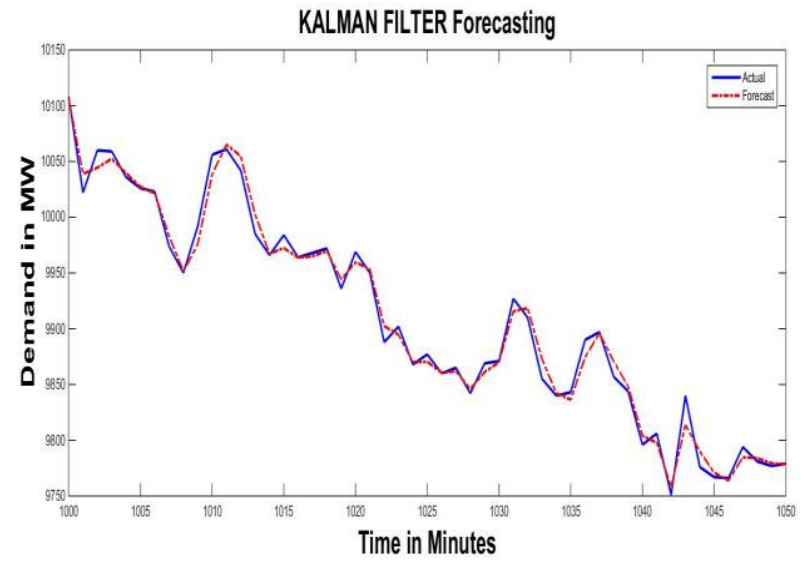

Fig (7). STLF using Kalman Filter zoomed plot

\section{RMSE}

RMSE represents the Root Mean Square Error used to calculate the accuracy between the actual and forecasted values. RMSE was calculated for both ARMA and Kalman Filter and the values are presented in Table1. The minimum RMSE value of 11.34 obtained for Kalman Filter indicates that, the model is best fit of the time series data compared to ARMA model.

$$
R M S E=\sqrt{\sum_{t=1}^{n}\left|\frac{\left(Y_{t}-\hat{Y}_{t}\right)^{2}}{n}\right|}
$$

\begin{tabular}{|l|l|c|}
\multicolumn{1}{c}{ TABLE I. RMSE CALCULATION } \\
\begin{tabular}{|c|l|c|}
\hline S.No & \multicolumn{1}{c|}{ Model } & RMSE \\
\hline 1 & ARMA & 31.1 \\
\hline 2 & KALMAN FILTER & 11.34 \\
\hline
\end{tabular}
\end{tabular}

\section{MAPE}

This measure represents the average absolute error occurred. It is independent of the scale of measurement, but affected by data transformation. MAPE does not panelize extreme 
deviations. Percentage of error is small for Kalman Filter when compared to ARMA model for the given time series.

$$
M A P E=\frac{1}{n} \sum_{t=1}^{n}\left|\frac{Y_{t}-\hat{Y}_{t}}{Y_{t}}\right| * 100
$$

TABLE II. MAPE CALCULATION
\begin{tabular}{|l|l|c|}
\hline S.No & \multicolumn{1}{c|}{ Model } & MAPE $\%$ \\
\hline 1 & ARMA & 0.23 \\
\hline 2 & KALMAN FILTER & 0.068 \\
\hline
\end{tabular}

\section{CONCLUSION}

The results indicated that Kalman Filter gives better load forecasting as compared to ARMA in terms of less measurement of error using Mean Absolute Percentage Error (MAPE). Kalman Filter algorithm is useful in real time prediction of demand forecast for next immediate hours. Actual demand information is captured through Supervisory Control and Data Acquisition (SCADA) system and will be used in Kalman Filter algorithm. During this process an enormous data change may generate from SCADA due to network failure, the telemeter value may not communicate to the server, due to failure of RTU or Transducers etc, due to major breakdown occurring in the system equipment and also may be due to any operational issues like choosing wrong system demand information as reference for forecast etc are some of the reasons. With this-reasons the SCADA may generate "Bad data" or "Wild points" in the system. The Kalman Filter may give wrong prediction demand information to the operator when bad data or wild points occur in data and leads to wrong operations performed by grid operator. This bad data needs to be filtered and recalculated with "Bad data detection algorithm" for better prediction. The Bad data detection and recalculation method need to be incorporated during Kalman Filter process which will produce better load forecast results to the Grid Operator.

\section{ACKNOWLEDGEMENT}

The authors greatly acknowledge Siddhartha Academy of General and Technical Education, Vijayawada for providing the facilities to carry out this research.

\section{REFERENCES}

[1] Samsul Ariffin, Abdul Karim and Saiful Azli Alwi, "Electricity load forecasting in UTP using moving averages and exponential smoothing Techniques," Applied Mathematical Sciences, Vol. 7, 2013.

[2] Vikram Veer Singh and Dr. Ajay Srivastava, "An introduction to load forecasting: Conventional and modern technologies," IRACST Engineering Science and Technology: An International Journal (ESTIJ), ISSN: 2250-3498 Vol.4, No. 2, April 2014.

[3] S.S. Vadhera, Power system Analysis and stability, Khanna Publishers Delhi, 2004.

[4] Box, G. E. P., G. M. Jenkins, and G. C. Reinsel, Time Series Analysis: Forecasting and Control, 3rd ed. Englewood Cliffs, NJ: Prentice Hall, 1994

[5] P. S. Moharir, "Predictive deconvolution (part-1 \& part-2)," IETE Journal of Research, vol. 2, issue: $4 \& 5,1985$

[6] C. Nataraja, M.B. Gorawar, G.N. Shilpa and J. Shri Harsha, "Shortterm load forecasting using time series analysis: A case study for Karnataka India," International Journal of Engineering Science and Innovative Technology, vol. 1, issue. 2, November 2012

[7] Mr. Dileshwar Prasad Patel, Associate Prof. Anshu.tiwari and Dr Vivek Dubey, "An analysis of short-term load forecasting by using time series analysis," International Journal of Research Computer and Communication Technology., vol. 2, issue: 2, Feb-2013

[8] Nima Amjady, "Short-term hourly load forecasting using time serie modeling with peak load estimation capability," IEEE Transaction on Power Systems, vol. 16, No. 3, August-2001.

[9] Shyh-Jier Huang and Kuang-Rong Shih, "Short-term load forecasting Via ARMA model identification including non-gaussian proces considerations," IEEE Transactions on Power Systems, Vol. 18, NO. 2, MAY 2003.

[10] V.Venkatesh, Shilpa G N, and Nataraja.C, "Study \& Development of short-term load forecasting models using stochastic time series analysis," International Journal of Engineering Research and Development, vol. 9, issue: 1, February 2014

[11] Steven M Kay, Fundamentals of Statistical Signal Processing: Estimation theory, volume 2, NJ: Prentice Hall, 1993.

[12] Anish Deb, Sunit K Sen, Gautam Sarkar and Asit K Datta, "Microprocessor based improved laod shedding controller (LSE) with kalman filter," IETE Journal of Research, vol. 41, issue: 4, 1995.

[13] Greg Welch and Gary Bishop, An Introduction to the Kalman Filter, Course 8, NC 27599-3175, 2001

[14] Stelios A. Markoulakis, George S. Stavrakakis And Triantafyllia G. Nikolaou, "Short-term load forecasting based on the Kalman filter and the neural-fuzzy network (ANFIS)," International Conference on Energy \& Environmental Systems, Chalkida, Greece, May 8-10, 2006.

[15] R. Shankar, K. Chatterjee and T.K. Chatterjee, "A very short-term load forecasting using Kalman filter for load frequency control with Economic Load Dispatch," Journal of Engineering Science and Technology Review, 5 (1) (2012).

[16] R.G. Brown, Introduction to Random Signal Analysis and Kalman Filtering, Wiley, New York, 1983. 